\title{
Imaging performance of a full-ring prototype PET-MRI system based on four-layer DOI-PET detectors integrated with a RF coil
} \author{
Taiga Yamaya ${ }^{1}$ \\ From PSMR 2015: 4th Conference on PET/MR and SPECT/MR \\ La Biodola, Isola d'Elba, Italy. 17-21 May 2015
}

Fumihiko Nishikido ${ }^{1 *}$, Hideaki Tashima', Mikio Suga ${ }^{2}$, Naoko Inadama$^{1}$, Yoshida Eiji', Takayuki Obata',

${ }^{1}$ National Institute of Radiological Sciences, Chiba, Japan
We are developing a PET system integrated with a birdcage RF-coil for PET-MRI in order to realize both high sensitivity and high spatial resolution of the PET image by using the 4-layered depth-of-interaction (DOI) PET detector. We constructed a fullring prototype system and evaluated performances, especially imaging performance, of the prototype system in simultaneous measurement. The prototype system consists of eight four-layer DOI-PET detectors and a prototype birdcage RF-coil developed for the proposed system. The PET detectors consist of six monolithic multi-pixel photon counter array (S11064-050P), a readout circuit, fourlayer DOI scintillator arrays and a shielding box made of $35 \mu \mathrm{m}$ thick copper foil. The crystal array consists of $2.0 \mathrm{~mm} \times$ $2.0 \mathrm{~mm} \times 5.0 \mathrm{~mm}$ LYSO crystals arranged in $38 \times 6 \times 4$ layer. The RF-coil has eight coil elements and the eight PET detectors are positioned at each element gap. The diameter of the RF-coil elements is $261 \mathrm{~mm}$. We conducted performance tests of the prototype system with a $3.0 \mathrm{~T}$ MRI (MAGNETOM Verio). Only the PET detectors, the RF-coil and the cables were in an MRI room during measurements. A data acquisition system and power supplies for the MPPCs and preamplifiers were outside the MRI room and connected to all the detectors through a penetration panel. As a result, the spatial resolutions of a Na-22 point source in the PET image were lower than $1.6 \mathrm{~mm}$ in whole the FOV due to the DOI capability. In addition, the influence of the simultaneous measurements on the PET performance is negligible. On the other hand, the SNR of the phantom image in the magnitude images was degraded from 259.7 to 209.4 due to noise contamination from the power supplies.

Authors' details

${ }^{1}$ National Institute of Radiological Sciences, Chiba, Japan. ${ }^{2}$ Chiba University, Chiba, Japan.

Published: 18 May 2015

doi:10.1186/2197-7364-2-S1-A18

Cite this article as: Nishikido et al:: Imaging performance of a full-ring prototype PET-MRI system based on four-layer DOI-PET detectors integrated with a RF coil. EJNMMI Physics 2015 2(Suppl 1):A18.

\section{SpringerOpen ${ }^{\circ}$}

(C) 2015 Nishikido et al; licensee Springer. This is an Open Access article distributed under the terms of the Creative Commons Attribution License (http://creativecommons.org/licenses/by/4.0), which permits unrestricted use, distribution, and reproduction in any medium, provided the original work is properly cited. 